\title{
Temozolomide Combined With Capecitabine In The Treatment Of Mixed Neuroendocrine Carcinoma Of The Lung With Poor Tolerance After Repeated Radiochemotherapy: A Case Report And Literature Review
}

This article was published in the following Dove Press journal:

OncoTargets and Therapy

\author{
Bin Zhang ${ }^{1,2, *}$ \\ Di Wang ${ }^{1, *}$ \\ Xia Zhang ${ }^{3}$ \\ Xiaonan Cui ${ }^{1}$ \\ Li Kong' \\ Minghuan $\mathrm{Li}^{2}$ \\ Jinming $\mathrm{Yu}^{2}$
}

'Department of Oncology, The First Affiliated Hospital of Dalian Medical University, Dalian, People's Republic of China; ${ }^{2}$ Shandong Cancer Hospital and Institute, Shandong First Medical University and Shandong Academy of Medical Sciences, Jinan, People's Republic of China; ${ }^{3}$ Department of Oncology, The Fifth People's Hospital of Dalian, Dalian, People's Republic of China

*These authors contributed equally to this work
Correspondence: Minghuan Li; Jinming Yu Shandong Cancer Hospital and Institute, Shandong First Medical University and Shandong Academy of Medical Sciences, No. 440, Yanzhou Road, Jinan 250II7,

People's Republic of China

Tel +8653 I-87984777

Fax +053I-87984079

Email Sy_Imh200I@I63.com;

sdyujinming@163.com

\begin{abstract}
The incidence of lung neuroendocrine carcinomas, which originate from lung neuroendocrine cells, is $1.35 / 100,000$, among which mixed neuroendocrine carcinomas are very rare. Because of the heterogeneity and significant differences in sensitivity to treatments, there is no effective treatment, and the prognosis is poor. In this article, we report the diagnosis and treatment of a case of mixed neuroendocrine carcinoma of the lung in our hospital. During the treatment, the patients had significant myelosuppression after initial chemotherapy, but benefited from oral chemotherapy consisting of a combination of capecitabine and temozolomide (CAPTEM). The report was approved by the affiliated Cancer Hospital of Shandong University.
\end{abstract}

Keywords: temozolomide, mixed neuroendocrine carcinoma, case report, poor tolerance, lung cancer, review

\section{Case Report}

The patient was a 65 -year-old male who developed paroxysmal cough without obvious causes in October 2013, with white sticky phlegm, accompanied by chest tightness and persistent back pain. On December 8, 2013, a chest CT showed a space-occupying lesion in the superior lobe of the left lung next to the mediastinum, which was located close to the aorta and showed significant enhancement on enhanced scan, with enlarged mediastinal lymph nodes in regions $1 \mathrm{~L}, 2 \mathrm{~L}$ and 5 , suggesting metastasis. On December 6, 2013, a left lung mass biopsy was performed under CT guidance. The pathology (biopsy of the left upper lung mass) and immunohistochemistry results were consistent with neuroendocrine carcinoma and small-cell carcinoma. Immunohistochemical staining showed $\mathrm{Syn}+, \mathrm{CgA}$ weak + , CK weak+, TTF-1+, broad-spectrum CK+, CK5/6-, P63 and Ki-67 (70-80\%) (Figure 1). Tumour maker determination results were as follows: neuron-specific enolase (NSE) $40.00 \mathrm{ng} / \mathrm{mL}$, cytokeratin-19 fragments (Cyfra21-1) $4.12 \mathrm{ng} / \mathrm{mL}$ and carcinoembryonic antigen (CEA) $28.10 \mathrm{ng} / \mathrm{mL}$. There was no obvious abnormality found in bone electroconvulsive therapy (ECT) and cranial MRI examination. The patient was diagnosed with left lung neuroendocrine carcinoma (small-cell type), 


\section{pathological picture and immunohistochemistry picture}

2013
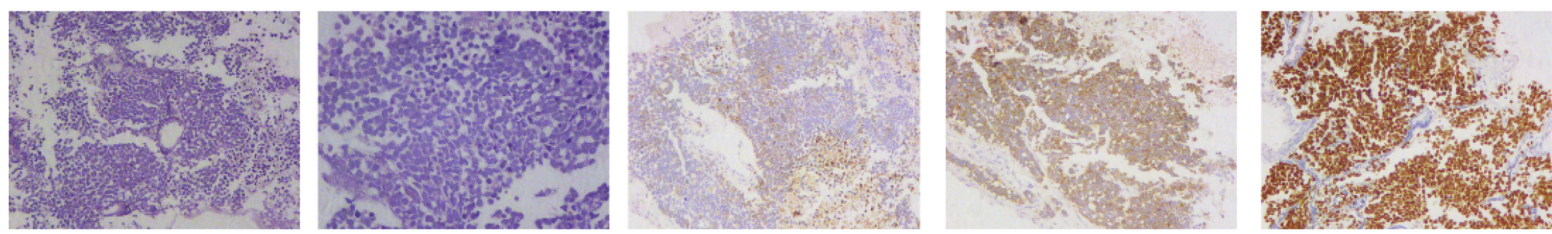

HE $(10 \times 10)$

HE (20X10)

CK7 (10X10)
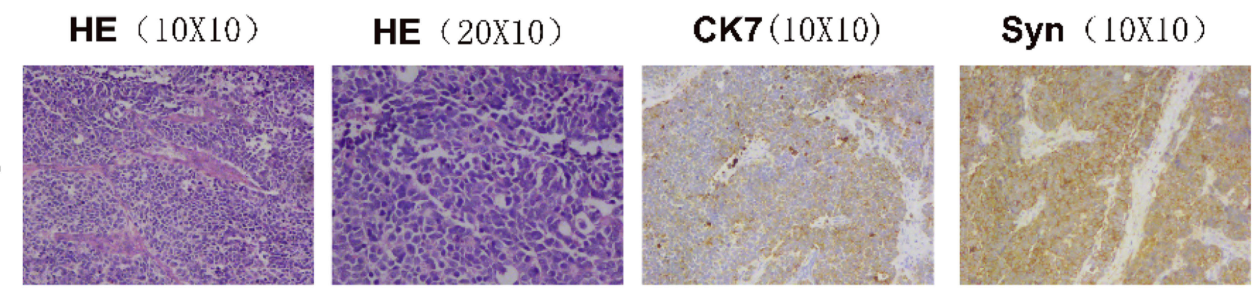

TTF-1 (10X10)

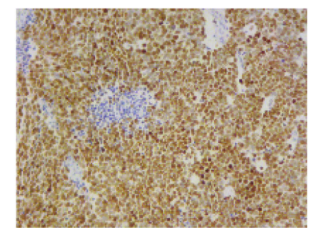

Figure I Patient's imaging pictures.

stage IIIB, cT4N2M0. An EP chemotherapy regimen was administered for four cycles. The first cycle consisted of VP-16 $0.1 \mathrm{~d} 1-5$, DDP $40 \mathrm{mg}$ d1-3 and q21d. After the first cycle of chemotherapy, degree IV granulocytopenia and degree II thrombocytopenia decreased, with $0.38 \times 10^{\wedge} 9 / \mathrm{L}$ neutrophils and $73 \times 10^{\wedge} 9 / \mathrm{L}$ platelets. Second-degree liver function damage occurred with $142 \mathrm{U} / \mathrm{L}$ glutamic-pyruvic transaminase and $67 \mathrm{U} / \mathrm{L}$ glutamic-oxal(o)acetic transaminase, and bilirubin was within the normal range. Granulocyte colony-stimulating factor (G-CSF) was given to increase the leukocyte count, and hepatoprotective support treatment was provided. The chemotherapy regimen was changed starting in the second cycle. The second to fourth cycles consisted of the following: VP-16 $0.1 \mathrm{~d} 1-4$, DDP $40 \mathrm{mg}$ d1-3 and q21d. After four cycles of chemotherapy, patient achieved partial response but fourth-degree bone marrow suppression were still present, and chemotherapy was stopped. Since March 14, 2014, the left lung lesion and primary tumour involving the mediastinal lymph node region were treated with radiotherapy consisting of DT $70 \mathrm{~Gy} / 35$ times. The treatment efficacy of radiotherapy resulted in almost complete response (CR), and the clinical symptoms disappeared. The patient was then followed up.

On August 11, 2014, the patient was admitted to the hospital for a follow-up assessment. Preventive brain irradiation was planned, and the $\mathrm{CT}$ examination showed no change in the pulmonary lesion. Two enlarged lymph nodes were found in the neck during the physical examination and were approximately $1.5 \mathrm{~cm} \times 1.0 \mathrm{~cm}$ in size. A lymph node biopsy showed mixed small-cell carcinoma and large-cell neuroendocrine carcinoma (Figure 1). The left supraclavicular metastatic lymph nodes were treated with radiotherapy, consisting of $60 \mathrm{~Gy} / 30 \mathrm{f}$, and with chemotherapy, consisting of paclitaxel $120 \mathrm{mg} \mathrm{d} 1$ and 8+DDP $40 \mathrm{mg} \mathrm{d} \mathrm{1-3}$ for two cycles. The side effects of chemotherapy were first-degree gastrointestinal reactions and second-degree granulocytopenia. Treatment efficacy of cervical lymph nodes after radiotherapy reached CR. In November 2014, the patient complained of pain in the waist. Abdominal CT showed that a soft tissue density nodule with a small diameter of approximately $2.5 \mathrm{~cm}$ was visible in the right costophrenic corner, which was close in proximity to the lumbar vertebrae. With the family's consent, the right costophrenic corner lymph nodes were treated with radiotherapy at DT $54 \mathrm{~Gy}$, and the patient reached PR. In February 2015, the patient complained of chest and back pain and a cough with a small amount of white sticky phlegm, with no chest tightness, chest pain, or haemoptysis. He also had lower back pain with a numerical rating scale (NRS) value of 3 and took acetaminophen tablets himself, resulting in an NRS decrease to 1 . On March 18, 2015, a follow-up exam showed an NSE value of $26.57 \mathrm{ng} / \mathrm{mL}$, Cyfra21 of $14.47 \mathrm{ng} / \mathrm{mL}$ and CEA of $8.18 \mathrm{ng} /$ $\mathrm{mL}$. CT revealed relapse at the original location and mediastinal lymph node metastasis, with no abnormality in the abdomen or head, and a bone scan showed no bone metastasis. Therefore, the patient was diagnosed with tumour relapse. The patient demonstrated poor tolerance to the previous chemotherapy, showing mainly bone marrow suppression and granulocytosis, and there is no standard treatment regimen for third-line chemotherapy. Thus, the treatment plan for this patient employed combined chemotherapy using oral medicine with low bone marrow toxicity, and the regimen consisted of temozolomide $\left(150 \mathrm{mg} / \mathrm{m}^{2} \mathrm{~d} 1-5, \mathrm{PO}\right)$ 
and capecitabine $\left(1000 \mathrm{mg} / \mathrm{m}^{2} \mathrm{~d} 1-14\right.$, PO and $\left.\mathrm{q} 21\right)$. The side effects were first-degree digestive tract reactions and seconddegree leukopenia. The blood count results were the following: white blood cells, $2.45-4.32 \times 10^{\wedge} 9 / \mathrm{L}$; neutrophils, 1.38 $2.68 \times 10^{\wedge} 9 / \mathrm{L}$; and platelets, $112-167 \times 10^{\wedge} 9 / \mathrm{L}$. The symptoms improved after oral administration of medicine to increase the white blood cell count, and the course of chemotherapy was not affected. Evaluation of the patient after two chemotherapy cycles revealed PR; however, CT examination after four cycles of treatment indicated disease progression. After consultation with internal medicine physicians, the patient was treated with pemetrexed 1.0. After one cycle, the symptoms were obviously aggravated. CT and tumour marker analysis suggested disease progression, and the treatment plan was changed to irinotecan combined with a cisplatin regimen for one cycle after exclusion of contraindications of chemotherapy. The exact plan was irinotecan (120 mg d1 and 8) and cisplatin (40 mg d1-3). After chemotherapy, the patient developed granulocytotic fever, abdominal pain, diarrhoea and watery stool, and the patient was treated with drugs to increase the white blood cell count, antibiotics, an antidiarrhoeal drug, nutritional support and other symptomatic and supportive treatments. The patient showed third- and fourth-degree suppression of platelets. Considering that the patient's general condition was poor, the patient was treated with a platelet transfusion, two transfusions of a therapeutic dose of machine-collected platelets from irradiated $\mathrm{B}$ type $\mathrm{Rh}$-positive blood and a transfusion of $200 \mathrm{~mL}$ of frozen plasma of virus-inactivated B type Rhpositive blood, and the fever and diarrhoea symptoms were improved. PD was still found in the second evaluation of the treatment efficacy, and due to the patient's poor general condition, the treatment was changed to single-drug chemotherapy with etoposide. The disease was not controlled, and the patient stopped treatment. The changes of CT, MRI and tumor marker of the patient are shown in Figure 2.

\section{Discussion}

Lung neuroendocrine tumours account for $20 \%$ of all primary lung tumours, which are classified by the World Health Organization (WHO) into low-grade malignant typical carcinoid, moderate malignant atypical carcinoid, highly malignant large-cell neuroendocrine carcinoma (LCNEC) and small-cell lung cancer (SCLC). Mixed LCNEC and SCLC neuroendocrine carcinomas are rare.

LCNEC accounts for $3 \%$ of lung cancers and has a strong tendency for metastasis. Early lobectomy can improve survival, but post-operative relapse is common. Most patients lose their surgery opportunity due to mediastinal lymph node metastasis or distant metastasis, and

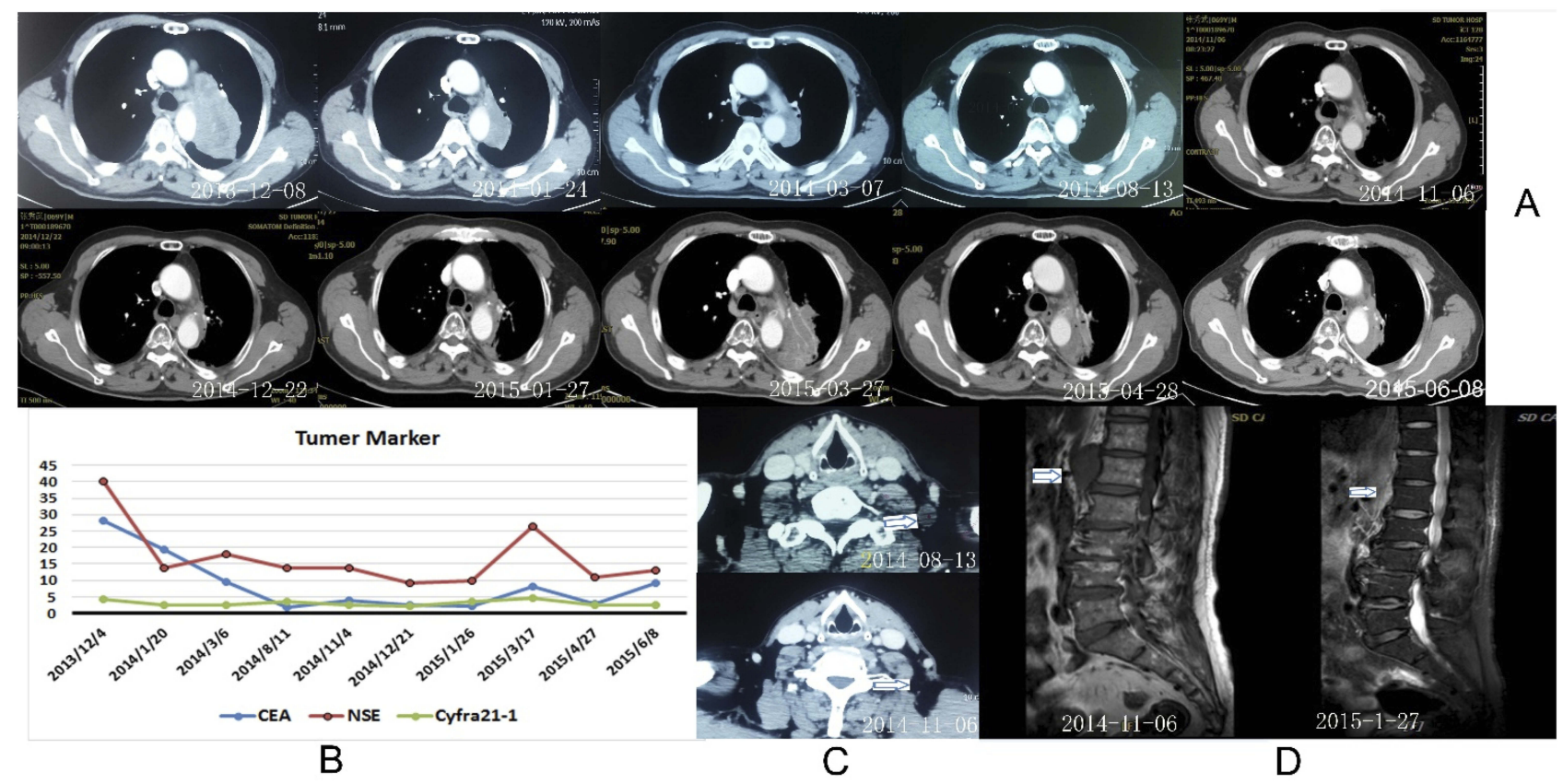

Figure 2 The above is the test result of the patient. (A). The figure shows the patient's chest enhancement CT from 20I3-I2-8 to 20I5-6-8, showing changes in primary lesions and mediastinal lymph node metastasis. (B). The figure shows the changes in tumor markers after the patient started treatment, especially after the application of the CAPTEM program in 2015-3-18, the NES decreased significantly, which suggests the superiority of CAPTEM. (C). CT shows the metastasis of cervical lymph nodes in patients with tumors. (D). In the figure, MRI shows the metastasis of the retroperitoneal lymph nodes in patients with tumors. 
the five-year survival rate is less than $40 \%$. The chemotherapy regimen for LCNEC is still controversial, while some studies noted that LCNEC treatment employing the SCLC chemotherapy regimen, such as etoposide or irinotecan, yielded a better survival rate than that associated with the LCNEC standard chemotherapy regimen. Although the National Comprehensive Cancer Network (NCCN) guidelines are still recommending treatment according to non-small-cell lung cancer (NSCLC), smallsample-size single-arm studies showed that the objective response rate (ORR) could reach $50 \%$ if the SCLC protocol is followed.

SCLC accounts for $14 \%$ of lung cancers. The median survival time is $8-12$ months, and the two-year survival rate is less than $10 \%$. Although SCLC is highly sensitive to initial chemotherapy and radiotherapy, it generally relapses and progresses within six months, and it generally has a poor response to second-line therapies, with a median survival time of only 4-5 months. The treatment efficacy may be highly dependent on the time between initial treatment and relapse. If the interval is less than three months, the efficacy of most drugs and regimens is poor, and if the interval is between three and six months, the expected efficacy rate is $25 \%$. If the interval is more than six months, the original regimen is recommended. ${ }^{1}$

This patient was initially diagnosed with a small-cell type of neuroendocrine carcinoma and was administered a first-line EP chemotherapy regimen. The patient showed poor tolerance, with fourth-degree bone marrow suppression appearing in the first cycle. The dose was reduced during the second cycle. Although this reduction affected treatment efficacy, at that time, no long-term effective drugs were available that could increase the white blood cell count as second-level prevention. Therefore, dosage reduction was imperative. Timely introduction of local radiotherapy is also a good choice for patients with intolerable chemotherapy side effects. In SCLC, radiotherapy intervention can improve local control. A phase III randomized controlled study by Jeremic ${ }^{2}$ showed that patients with ED-SCLC with metastatic lesions reaching CR and chest lesions reaching CR or PR after chemotherapy could significantly benefit from chest radiotherapy. The median survival time was extended by half a year, and the fiveyear survival rate was $9 \%$. The subsequently initiated multicentre randomized controlled study (CREST) was reported at the American Society of Clinical Oncology (ASCO) and American Society for Radiation Oncology (ASTRO) conferences in 2014. The results showed that thoracic radiation treatment (TRT) reduced the intrathoracic relapse rate by nearly $50 \%$ compared with that in the control group and significantly decreased the progressionfree survival (PFS) time ( $\mathrm{HR}=0.73$ ). When followed up to two years, the survival of the TRT group was significantly better than that of the control group $(13 \%$ vs. $3 \%$, $\mathrm{p}=0.004)$. In terms of LCNEC, a clinical study ${ }^{3}$ validated the efficacy of gamma knife radiosurgery (GKRS) for the treatment of LCNEC patients with brain metastasis and reported a one-year neurological death-free rate of $93 \%$ and a PFS rate of $87 \%$ among 101 patients. Another study ${ }^{4}$ also found that the median PFS and overall survival improved in LCNEC patients receiving TRT (12.5 vs. 5 months, $\mathrm{p}=0.02$, and 28.3 vs. 5 months, $\mathrm{p}=0.004$, respectively). The above data confirm the positive effects of local radiotherapy.

There are many choices of treatment plans, including somatostatin synthesis analogues such as octreotide and lanreotide, platinum-type drugs, etoposide, temozolomide, capecitabine and other cytotoxic drugs, which can be used in the treatment of neuroendocrine carcinoma. This patient had very poor tolerance to chemotherapy, and choosing a drug that could be tolerated was the key to successful treatment. For patients with bronchopulmonary or thymic carcinomas with a low or moderate tumour load and obvious symptoms, Temozolomide treatment, either alone or in combination with octreotide or lanreotide, is an option for addressing the tumour load and any associated symptoms. ${ }^{5,6}$ The CAPTEM combination has been used in neuroendocrine carcinoma. The regimen has high activity and good tolerance and can prolong the survival time of patients with well-differentiated metastatic neuroendocrine tumours. ${ }^{7}$ A retrospective analysis indicated that the objective remission rate associated with this combined regimen was $70 \%$, and the median PFS was 18 months. Another retrospective study reported that the remission rate of 18 patients was $61 \%$, and one patient had achieved complete pathological remission as demonstrated by surgery. ${ }^{8-10}$ Cives $^{11}$ also mentioned that CAPTEM chemotherapy promoted the prolongation of PFS in patients with pancreatic neuroendocrine tumours. In 2018, a meta-analysis of the safety and efficacy of the CAPTEM regimen in the treatment of advanced neuroendocrine tumours ${ }^{12}$ showed that most of the 384 patients included in the study had a median total survival of more than 12 months, and the median PFS was similar to or slightly higher than that of other therapies, suggesting that CAPTEM is effective and relatively safe in the treatment of advanced neuroendocrine neoplasm (NEN) 
patients. In this case, the patient had poor tolerance to and obvious bone marrow suppression from the first-line EP regimen, while the degree of bone marrow suppression decreased from fourth degree to second degree after application of the CAPTEM regimen. The CAPTEM regimen not only alleviated side effects but also prolonged the course of chemotherapy and improved the patient's life quality, which are important advantages of the CAPTEM regimen. The patient obtained nearly 3 months of PFS from the application of this regimen, which is superior than the PFS afforded by other protocols.

If disease progression relapses after CAPTEM chemotherapy, what treatment method can be used? Recently, immunotherapy has entered the public view. A cytotoxic $\mathrm{T}$ lymphocyte-associated protein-4 (CTLA-4) inhibitor, ipilimumab, is the first immune target drug used for SCLC. In the exploratory analysis of phase II clinical trials of combined chemotherapy using ipilimumab, ${ }^{13}$ it was found that patients with a high expression of antibodies before treatment may benefit from this regimen. A meta-analysis ${ }^{14}$ indicated that ipilimumab improved the PFS (six months: $\mathrm{RR}=1.16, \mathrm{P}=0.02$; one year: $\mathrm{RR}=1.39, \mathrm{P}=0.02$ ) and six-month immunerelated PFS (irPFS) $(\mathrm{RR}=1.60, \mathrm{P}=0.004)$ in $1084 \mathrm{SCLC}$ patients. However, because of the addition of ipilimumab, immune-related toxicity was more obvious in the immunochemotherapy group. Another significant breakthrough was the discovery that blocking the binding of programmed death protein 1 (PD-1) to its ligand (PD-L1) could inhibit the immune escape of tumour cells from $\mathrm{T}$ cells. ${ }^{15}$ Schachter ${ }^{16}$ reported the therapeutic effects of antiPD-1/PD-L1 drugs on tumours using SCLC as an example disease. Grabowski found that PD-L1 was expressed in $100 \%$ of poorly differentiated neuroendocrine carcinomas, and the expression rate of PD-L1 in well-differentiated neuroendocrine tumours was also as high as $50 \%$. The PD-1 inhibitor nivolumab combined with the CTLA-4 inhibitor ipilimumab has become a second-line therapy for advanced SCLCs after chemotherapy. ${ }^{17}$ The results of the clinical trial CheckMate-032 showed that regardless of the expression level of PD-L1 and whether the cancer was sensitive to the platinum-type chemotherapeutic drugs used in first-line treatments, the effects of immunotherapy were remarkable and could last for a longer period. The two-year survival rate of the combined treatment group reached $30 \%$. However, systematic studies on LCNEC or mixed neuroendocrine carcinoma are lacking, and no related drugs are on the market in China at this time.
To date, no study has shown the efficacy of targeted therapy in mixed neuroendocrine carcinoma. One possible target is the somatostatin receptor. Somatostatin analogues can effectively control symptoms and prolong survival in LCNEC patients with positive somatostatin imaging. ${ }^{18}$ Other targets include IGF-1R, the Hh pathway, the Notch pathway, the $\mathrm{P} 13 \mathrm{~K} / \mathrm{AKT} / \mathrm{mTOR}$ pathway, the Bcl-2 family of antiapoptotic proteins, PARP and VEGF. Everolimus, an mTOR inhibitor, is also one recommended drug in NCCN guidelines. In a phase 3 RADIANT-4 clinical trial, ${ }^{19} 302$ patients with advanced, progressive, well-differentiated, nonfunctioning lung or gastrointestinal neuroendocrine tumours were included. The results showed that the median PFS was 11.0 months (95\% CI 9.2-13.3) in the treatment group and 3.9 months (3.6-7.4) in the placebo group. At a recent Gastrointestinal Cancers Symposium, ${ }^{20}$ experts also pointed out that everolimus reduced the risk of disease progression by $40 \%$ compared with the placebo group and showed the potential to block cancer cells from growing.

Unfortunately, the patient in this study did not have an opportunity to wait for the development and application of new drugs; however, his case confirmed the efficacy and safety of CAPTEM, which can be used in the treatment of neuroendocrine tumours. We hope that through this article, clinicians will have a better understanding of mixed neuroendocrine carcinoma.

\section{Statement Of Patient's Family}

The patient's next of kin have provided written informed consent for the case details to be published.

\section{Disclosure}

The authors report no conflicts of interest in this work.

\section{References}

1. Asamura H, Chansky K, Crowley J, et al. The international association for the study of lung cancer lung cancer staging project: proposals for the revision of the $\mathrm{n}$ descriptors in the forthcoming 8th edition of the TNM classification for lung cancer. $J$ Thorac Oncol. 2015;10:16751684. doi:10.1097/JTO.0000000000000678

2. Prelaj A, Rebuzzi SE, Del Bene G, et al. Evaluation of the efficacy of cisplatin-etoposide and the role of thoracic radiotherapy and prophylactic cranial irradiation in LCNEC. ERJ Open Res. 2017;3. doi:10.1183/23120541.00128-2016

3. Jeremic B, Shibamoto Y, Nikolic N, et al. Role of radiation therapy in the combined-modality treatment of patients with extensive disease small-cell lung cancer: a randomized study. $J$ Clin Oncol. 1999;17:2092-2099. doi:10.1200/JCO.1999.17.7.2092

4. Kawabe T, Yamamoto M, Sato Y, et al. Gamma Knife radiosurgery for brain metastases from pulmonary large cell neuroendocrine carcinoma: a Japanese multi-institutional cooperative study (JLGK1401). $J$ Neurosurg. 2016;125:11-17. doi:10.3171/2016.7.GKS161459 
5. Cives M, Strosberg JR. Gastroenteropancreatic neuroendocrine tumors. CA Cancer J Clin. 2018;68:471-487. doi:10.3322/caac.v68.6

6. Lu Y, Zhao Z, Wang J, et al. Safety and efficacy of combining capecitabine and temozolomide (CAPTEM) to treat advanced neuroendocrine neoplasms: a meta-analysis. Medicine (Baltimore). 2018;97:e12784. doi:10.1097/MD.0000000000012784

7. Chong CR, Wirth LJ, Nishino M, et al. Chemotherapy for locally advanced and metastatic pulmonary carcinoid tumors. Lung Cancer. 2014;86:241-246. doi:10.1016/j.lungcan.2014.08.012

8. Crona J, Fanola I, Lindholm DP, et al. Effect of temozolomide in patients with metastatic bronchial carcinoids. Neuroendocrinology. 2013;98:151-155. doi:10.1159/000354760

9. Fine RL, Gulati AP, Krantz BA, et al. Capecitabine and temozolomide (CAPTEM) for metastatic, well-differentiated neuroendocrine cancers: the pancreas center at Columbia University experience. Cancer Chemother Pharmacol. 2013;71:663-670. doi:10.1007/s00280-0122055-z

10. Ekeblad S, Sundin A, Janson ET, et al. Temozolomide as monotherapy is effective in treatment of advanced malignant neuroendocrine tumors. Clin Cancer Res. 2007;13:2986-2991. doi:10.1158/10780432.CCR-06-2053

11. Chan JA, Stuart K, Earle CC, et al. Prospective study of bevacizumab plus temozolomide in patients with advanced neuroendocrine tumors. J Clin Oncol. 2012;30:2963-2968. doi:10.1200/JCO.2011.40.3147

12. Strosberg JR, Fine RL, Choi J, et al. First-line chemotherapy with capecitabine and temozolomide in patients with metastatic pancreatic endocrine carcinomas. Cancer. 2011;117:268-275. doi:10.1002/cncr.v117.2

13. Arriola E, Wheater M, Galea I, et al. Outcome and biomarker analysis from a multicenter Phase 2 study of ipilimumab in combination with carboplatin and etoposide as first-line therapy for extensive-stage SCLC. J Thorac Oncol. 2016;11:1511-1521. doi:10.1016/j.jtho.2016.05.028
14. Zhang H, Shen J, Yi L, Zhang W, Luo P, Zhang J. Efficacy and safety of ipilimumab plus chemotherapy for advanced lung cancer: a systematic review and meta-analysis. J Cancer. 2018;9:4556-4567. doi: $10.7150 /$ jca. 27368

15. Dong H, Strome SE, Salomao DR, et al. Tumor-associated B7-H1 promotes T-cell apoptosis: a potential mechanism of immune evasion. Nat Med. 2002;8:793-800. doi:10.1038/nm730

16. Schachter J, Ribas A, Long GV, et al. Pembrolizumab versus ipilimumab for advanced melanoma: final overall survival results of a multicentre, randomised, open-label phase 3 study (KEYNOTE-006). Lancet. 2017;390:1853-1862. doi:10.1016/ S0140-6736(17)31601-X

17. Wolchok JD, Chiarion-Sileni V, Gonzalez R, et al. Overall survival with combined nivolumab and ipilimumab in advanced melanoma. $N$ Engl J Med. 2017;377:1345-1356. doi:10.1056/NEJM oa1709684

18. Filosso PL, Ruffini E, Oliaro A, et al. Large-cell neuroendocrine carcinoma of the lung: a clinicopathologic study of eighteen cases and the efficacy of adjuvant treatment with octreotide. $J$ Thorac Cardiovasc Surg. 2005;129:819-824. doi:10.1016/j.jtcvs. 2004.05.023

19. Yao JC, Fazio N, Singh S, et al. Everolimus for the treatment of advanced, non-functional neuroendocrine tumours of the lung or gastrointestinal tract (RADIANT-4): a randomised, placebo-controlled, phase 3 study. Lancet. 2016;387:968-977. doi:10.1016/ S0140-6736(15)00817-X

20. Singh S, Asa SL, Dey C, et al. Diagnosis and management of gastrointestinal neuroendocrine tumors: an evidence-based Canadian consensus. Cancer Treat Rev. 2016;47:32-45. doi:10.1016/j.ctrv. 2016.05.003
OncoTargets and Therapy

\section{Publish your work in this journal}

OncoTargets and Therapy is an international, peer-reviewed, open access journal focusing on the pathological basis of all cancers, potential targets for therapy and treatment protocols employed to improve the management of cancer patients. The journal also focuses on the impact of management programs and new therapeutic

\section{Dovepress}

agents and protocols on patient perspectives such as quality of life, adherence and satisfaction. The manuscript management system is completely online and includes a very quick and fair peer-review system, which is all easy to use. Visit http://www.dovepress.com/ testimonials.php to read real quotes from published authors. 